\title{
DEPREDACIÓN DE TURBO CASTANEA (TROCHIDA: TURBINIDAE) POR FASCIOLARIA TULIPA (NEOGASTROPODA: FASCIOLARIIDAE) \\ EN CUBA
}

\section{Depredation of Turbo castanea (Trochida: Turbinidae) by Fasciolaria tulipa (Neogastropoda: Fasciolariidae) in Cuba}

\begin{abstract}
Asiel Cabrera Guerrero ${ }^{1, *}$, Rogelio Muñoz Li $^{2}$ y Flavia Álvarez Denis ${ }^{3}$
1, 2, 3 Grupo Científico Estudiantil de Ecología Marina "Dra. María Elena Tbarra Martín”. Departamento de Biología y Geografía, Facultad de Ciencias Naturales y Exactas, Universidad de Oriente. ${ }^{1}$ (D) orcid.org/0000-0002-8858-0331. ${ }^{2}$ (i) orcid.org/0000-0003-3549-5560. ${ }^{3}$ (b orcid.org/0000-0002-3732-3693.

*Para correspondencia: asiel.cabrera@estudiantes.uo.edu.cu.
\end{abstract}

\section{RESUMEN}

El Tulipán Verdadero, Fasciolaria tulipa (Linnaeus, 1758), es un depredador de gasterópodos y bivalvos marinos, En el presente trabajo se registra por primera vez para Cuba la depredación de Turbo castanea (Gmelin, 1791) por F. tulipa. El registro se documenta fotográficamente en el sublitoral de la playa de Sardinero en Santiago de Cuba.

Palabras clave: Tulipán verdadero, Turbante castaño, alimentación, Caribe.

\section{ABSTRACT}

The True Tulip, Fasciolaria tulipa (Linnaeus, 1758), is a predator of marine gastropods and bivalves. In this work, the predation of Turbo castanea (Gmelin, 1791) by F. tulipa is recorded for the first time in Cuba. The record is photographed in the sub-littoral of Sardinero beach in Santiago de Cuba.

Keywords: True tulip, chestnut turban, feeding, Caribe.

El neogasterópodo Fasciolaria tulipa (Linnaeus, 1758) es de hábitats marinos y estuarinos. Se encuentra ampliamente distribuido desde Carolina del Norte hasta Texas (EEUU), gran parte del Caribe hasta Brasil. Habita en praderas de angiospermas marinas y fondos arenosos-coralinos, entre la zona intermareal y aproximadamente a $73 \mathrm{~m}$ de profundidad (Santhanam, 2019). Por su parte, Turbo castanea Gmelin, 1791 comparte la distribución de F. tulipa y es frecuente encontrarlos sobre fondos de sustratos no consolidados, lechos de algas y angiospermas marinas en un rango batimétrico entre cero y $141 \mathrm{~m}$ (Wehrtmann y Cortés, 2009). En Cuba, han sido registradas en varias localidades y en hallazgos arqueológicos (De la Guardia et al., 2003; Diez y Jover, 2012; 2013; Orihuela y Jiménez, 2017).

El día 8 de mayo del 2019, a las 14:00 horas, en la playa de Sardinero (1957'34.59' ' N; $75^{\circ} 47^{\prime} 00.92^{\prime \prime}$ O), al sureste de la provincia de Santiago de Cuba (Fig. 1), se observó un ejemplar de F. tulipa devorando un individuo de T. castanea (Fig. 2) en una pradera dominada por Thalassia testudinum Banks ex König con parches de Syringodium filiforme Kützing. La observación se realizó a través buceo libre con equipo ligero a una profundidad de $4 \mathrm{~m}$ y a $13 \mathrm{~m}$ de la orilla. El proceso observado duró alrededor de dos horas y media y al final se observó la concha de $T$. castanea vacía, mientras que el ejemplar de $F$. tulipa permaneció en reposo con el opérculo cerrado durante un tiempo prolongado. 
La especie $F$. tulipa es un depredador activo de hábito carnívoro y su dieta consiste fundamentalmente de otros moluscos, como bivalvos y gasterópodos. Aunque los gasterópodos de gran tamaño son los más frecuentes durante su alimentación (Paine, 1963). La depredación de T. castanea por F. tulipa ha sido consignada para los cayos de la Florida en ecosistemas de T. testudinum (Paine, 1963; Engstrom, 1982). La presente observación confirma la coexistencia de estos dos gasterópodos en las costas de Cuba oriental como ha sido documentado por Diez y Jover $(2012,2013)$ y constituye la primera evidencia del uso de $T$. castanea como recurso alimenticio de F. tulipa.

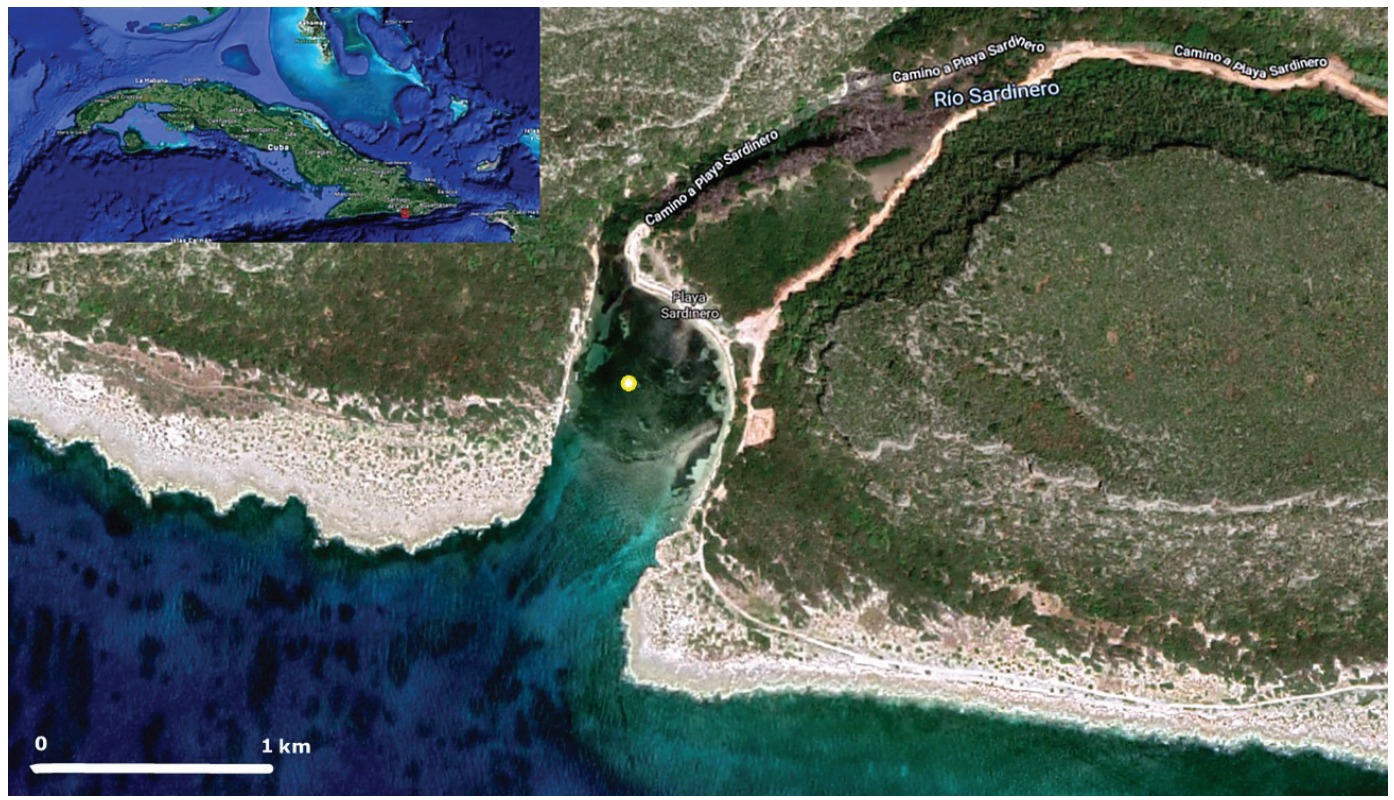

Figura 1. Ubicación geográfica de la playa Sardinero. El circulo amarillo representa la localidad donde se realizó la observación.

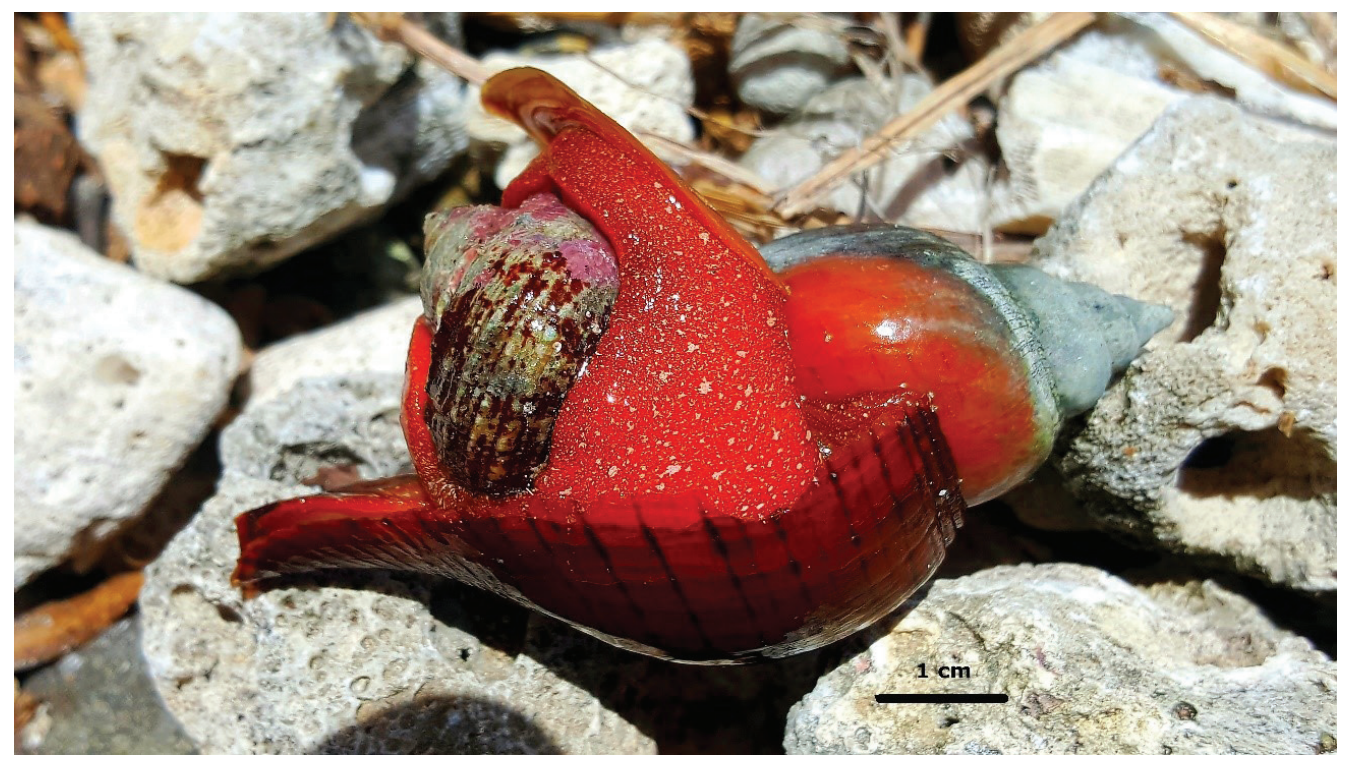

Figura 2. Depredación de Turbo castanea por Fasciolaria tulipa en playa Sardinero, Cuba. 


\section{AGRADECIMIENTOS}

A Yander L. Diez García, por la legitimación taxonómica de las dos especies de moluscos. Agradecemos a los revisores y a Abdiel Jover Capote por su sugerencias y comentarios.

\section{LITERATURA CITADA}

De la Guardia, E., G. González-Sansón y C. Aguilar. 2003. Biodiversidad marina en la laguna costera el Guanal, Cayo Largo, Cuba. Revista de Investigaciones Marinas, 24 (2): 111-116.

Diez, Y. L. y A. Jover. 2012. Moluscos marinos del sector Bahía de Puerto Padre-Bahía de Nipe, Cuba. Amici Molluscarum, 20 (1): 17-28.

Diez, Y. L. y A. Jover. 2013. Lista y distribución de los moluscos marinos de Santiago de Cuba, costa suroriental de Cuba. Amici Molluscarum, 21 (1): 23-38.

Engstrom, N. A. 1982. Escape responses of Turbo custanea to the predatory gastropod Fasciolaria tulipa. Veligev, 25: 163-168.

Orihuela, J. y O. Jiménez. 2017. Reporte del molusco marino Busycon perversum (Gastropoda: Busyconidae) del sitio arqueológico El Morrillo, Matanzas, Cuba. Cuba Arqueológica, 10 (1): 52-59.

Paine, R. 1963. Trophic relationships of 8 sympatric predatory gastropods. Ecology, 44 (1): 63-73.

Santhanam, R. 2019. Biology and ecology of edible marine gastropod molluscs. Apple Academic Press, Inc. Waretown, New Jersy, USA, 459 pp.

Wehrtmann, I. y J. Cortés, 2009. Marine biodiversity of Costa Rica, Central America. Springer, Dordrecht, Nederland, 538 pp.

[Recibido: 19 de mayo, 2019. Aceptado para publicación: 15 de septiembre, 2019] 